\title{
Evaluation of a Minimum Power Handoff Algorithm
}

\author{
Chen-Nee Chuah \\ chuah@winlab.rutgers.eduryates@ece.rutgers.edu
Wireless Information Networks Laboratory (WINLAB)
Dept. of Electrical and Computer Engineering
Rutgers University
PO Box 909, Piscataway, NJ 08855

\begin{abstract}
Previous work has shown that a handoff algorithm based on SIR alone (SIR Based Handoff) prohibits handoffs near nominal cell boundaries, causing cell dragging and unnecessarily high transmitter powers. In this paper, we propose the Minimum Power Handoff (MPH) algorithm in which mobiles constantly search for a combination of base and channel assignment that minimizes the uplink transmitted power. This algorithm is shown to reduce the average power level by $4 \mathrm{~dB}$ compared to SIR Based Handoff. Results show that using received power as a handoff criterion reduces call dropping but increases the number of unnecessary handoffs significantly. To avoid a "ping pong" effect, a timer is introduced to delay the intercell handoff.
\end{abstract}

\section{Introduction}

The radio propagation between a mobile and its serving base station is constantly changing due to user motion, giving rise to the need for power control, handoff, and channel reassignment (also known as intra-cell handoffs). These radio resource management tasks have been studied extensively in [1-13].

Recent work addresses the joint optimization of two or more tasks such as power control and base station assignment in [14-16]. For single channel CDMA systems, these works verify the optimality of minimum transmitter power as a criterion for handoff. In this work, we examine the effectiveness of transmitter power minimization as part of an integrated resource management strategy in the context of a multichannel system.

An SIR Based Handoff (SIRBH) algorithm was proposed in [17] for a power controlled multichannel system. In this system, each user aims for a target SIR $\gamma_{t}$ and handoffs are made when the absolute SIR drops below a certain threshold, $\gamma_{\text {ho }}$, which is normally less than $\gamma_{t}$. As pointed out in [17], SIRBH causes call dragging and inhibits handoffs near geographical cell boundaries. This provides a motiva- tion to study the use of transmitted power as an alternative handoff criterion. As we shall see, shadow fading causes unnecessary handoffs under the MPH algorithm. A timer is then introduced to mitigate this effect.

\section{Radio Resource Allocation}

In this section, we will describe the elements of a radio resource allocation strategy that includes power control, handoff, and call admission. Let $p_{i}$ denote the power transmitted by mobile $i$ and $G_{i j}$ the gain factor from mobile $i$ to base station $j$ due to both path loss and shadow fading. The interference plus noise seen by mobile $i$ measured at base station $j$ on channel $k$ is

$$
I_{i j}^{(k)}=\sum_{\substack{i^{\prime} \in M_{k} \\ i^{\prime} \neq i}} p_{i^{\prime}} G_{i^{\prime} j}+\eta
$$

where $M_{k}$ is the set of mobiles that are assigned to channel $k$ and $\eta$ is the base station receiver noise. The uplink SIR of mobile $i$ at base $j$ on channel $k$ is

$$
\gamma_{i j}^{(k)}=\frac{p_{i} G_{i j}}{I_{i j}^{(k)}}
$$

\section{$2.1 \quad$ Power Control}

At all times, the transmitted power $p_{i}$ is adjusted to attain target SIR, $\gamma_{t}$, subject to a maximum power contraint as in [18], via

$$
p_{i}=\min \left\{p_{\max }, \gamma_{t} I_{i j}^{(k)} / G_{i j}\right\}
$$

After the handoff and power control algorithms are executed, the quality of the call is checked against the minimum SIR requirement. The call will be dropped if its SIR falls below $\gamma_{\text {drop }}$. 


\subsection{Minimum Power Handoff}

If a mobile $i$ is assigned to base $j_{0}$ on channel $k_{0}$, the mobile will run the MPH algorithm every second to look for the base station $j^{*}$ and channel $k_{i}^{*}\left(j^{*}\right)$, that allows $\gamma_{t}$ to be achieved with the minimum transmitted power. For a given mobile $i$ and base station $j$, the minimum power channel $k_{i}^{*}(j)$ satisfies

$$
k_{i}^{*}(j)=\arg \min _{k} I_{i j}^{(k)}
$$

Thus, the base that requires the minimum power is

$$
j^{*}=\arg \min _{j} \gamma_{t} \frac{I_{i j}^{\left(k_{i}^{*}(j)\right)}}{G_{i j}}
$$

If the best base $j^{*}$ is not the current base $j_{0}$, an intercell handoff is made. If the current base is still the best but the current channel no longer offers the minimum interference, $j^{*}=j_{0}$ but $k^{*} \neq k_{0}$, intracell handoff is made by switching the connection from channel $k_{0}$ to $k^{*}$. Otherwise, the call remains assigned to the current base station and channel.

\subsection{The Handoff Timer, MPHT}

To reduce the number of unnecessary handoffs, a conditional timer is introduced to delay handoff requests. This results in a modified algorithm, MPHT. Whenever the best base station differs from the current one, $j^{*} \neq j_{0}$, the maximum achievable SIR at the current base station is estimated as

$$
\gamma_{\max }=p_{\max } G_{i j} / I_{i j}^{(k)}
$$

Note that $\gamma_{\max }$ is only an estimate of the maximum achievable SIR since it neglects the response by other mobiles to a maximum power transmission. If $\gamma_{\max }$ is less than the minimum required SIR, $\gamma_{\text {drop }}$, the timer will not be invoked and handoffs are allowed to take place immediately if the other rules for intercell handoffs are satisfied. Otherwise, the timer is set.

The timer will be incremented each time if the condition $j^{*} \neq j_{0}$ is true for the next consecutive second; otherwise it is reset. The intercell handoffs are delayed until the timer reaches a specific count, denoted by $T_{H}$. When this happens, the call will be handed over to the appropriate base station. The timer is turned off after the handoff succeeds. If the timer value is less than $T_{H}$, no intercell handoffs will be made and the particular call will still be carried by the old base station with the power being adjusted accordingly.

In cases where intercell handoffs fail or are delayed by the timer, intra-cell handoffs will be made to minimize the transmitted power, and hence co-channel interference.

\subsection{Call Admission}

We assume each base transmits a beacon signal on a separate channel. When a new mobile $i$ arrives, it is assigned to the base with the strongest beacon signal. After mobile $i$ is assigned to base $j$, a minimum interference scheme is used to assign a channel $k$ satisfying

$$
I_{i j}^{(k)}=\min _{k^{\prime} \in C_{j}}\left\{I_{i j}^{\left(k^{\prime}\right)}\right\}
$$

where $C_{j}$ is the set of free channels at base station $j$.

We consider an admission control based on SIR threshold, $\gamma_{\text {new }}$, as described in [17]. A new call is accepted only if the assigned channel $k$ can provide an estimated maximum SIR $\gamma_{\max }$, as defined by (6) not less than $\gamma_{\text {new }}$; otherwise, the new call is blocked.

\section{Simulation Model}

The performance of MPH and MPHT in a channelized system was studied by simulation on a one dimensional cellular system. Both uplink and downlink power control are implemented to attain a common SIR $\gamma_{t}$ for all terminals in the system. A mobile $i$ assigned to base station $k$ adjusts its power $p_{i}$ every second via (3). If at any time the SIR of a call falls below the minimum SIR requirement $\gamma_{\text {drop }}$, the call is dropped.

In our simulation, $B=20$ base stations are spaced uniformly 2000 meters apart on a ring to avoid edge effects. The new call arrival process is an independent Poisson process with mean arrival rate $\lambda$ calls per second, uniformly distributed over the entire ring. Call durations are independent exponential random variables with mean $1 / \mu=120$ s. A mobile's speed follows a truncated Gaussian distribution with mean speed of $90 \mathrm{~km} / \mathrm{hr}$, minimum speed of $60 \mathrm{~km} / \mathrm{hr}$ and maximum speed of $120 \mathrm{~km} / \mathrm{hr}$. The "clockwise" and "counterclockwise" directions are equally likely. The velocity of a terminal remains fixed throughout duration of a call. A set of $M=40$ channels are available. With $B$ base stations, the normalized traffic load is $\rho=\lambda /(\mu M B)$ erlangs $/$ channel/cell.

The radio link gain includes both a propagation loss of order $\alpha=4$ and lognormal fading. The link gain $G_{i j}$ from mobile $i$ at a distance $d_{i j}$ to base $j$ in units of $\mathrm{dB}$ is

$$
10 \log G_{i j}=-10 \alpha \log d_{i j}+S_{j}\left(d_{i j}\right)
$$

where $S_{j}\left(d_{i j}\right)$, the position dependent shadow fading factor, is modeled as a zero mean Gaussian random variable with standard deviation $\sigma=6 \mathrm{~dB}$. It is assumed that the shadow fading has a distance autocorrelation function

$$
\rho(d)=E\left[S_{j}\left(d^{\prime}\right) S_{j}\left(d^{\prime}+d\right)\right]=e^{-|d| / d_{0}}
$$

with a correlation distance, $d_{0}$ of $45 \mathrm{~m}$. Shadow fading measurements are obtained at $\delta=5 \mathrm{~m}$ intervals and the shadow fading proceses $S_{j}(d)$, and $S_{j^{\prime}}\left(d^{\prime}\right)$ for distinct base stations $j$ and $j^{\prime}$ are assumed to be independent. 


\begin{tabular}{|c|l|l|}
\hline$B$ & No. of base stations & 20 \\
\hline$M$ & No. of channels in system & 40 \\
\hline$D$ & Distance between 2 base stations & $2000 \mathrm{~m}$ \\
\hline$\alpha$ & Propagation exponent & 4 \\
\hline$\sigma$ & Std. deviation of lognormal fading & $6 \mathrm{~dB}$ \\
\hline$d_{0}$ & Shadow Fading Correlation Distance & $45 \mathrm{~m}$ \\
\hline$\delta$ & Sampling Distance & $5 \mathrm{~m}$ \\
\hline$v_{\min }$ & Minimum Speed & $60 \mathrm{~km} / \mathrm{hr}$ \\
\hline$v_{\max }$ & Maximum Speed & $120 \mathrm{~km} / \mathrm{hr}$ \\
\hline $\bar{V}$ & Mean Speed & $90 \mathrm{~km} / \mathrm{hr}$ \\
\hline$\eta$ & Receiver Noise Power & $-150 \mathrm{dBm}$ \\
\hline$p_{\max }$ & Maximum Transmitter Power & $0 \mathrm{dBw}$ \\
\hline$\gamma_{\text {drop }}$ & Floor SIR & $16 \mathrm{~dB}$ \\
\hline$\gamma_{t}$ & Target SIR & $19 \mathrm{~dB}$ \\
\hline$\gamma_{\text {new }}$ & New Call SIR Threshold & $22 \mathrm{~dB}$ \\
\hline$T$ & Intercell Handoff Timer & 1 \\
\hline
\end{tabular}

Table 1: Simulation Parameters

Users are updated asynchronously every second and resource adjustments including transmitted power adjustment, handoff algorithm and service quality checking are performed. Positions of all users are updated every second, after which the interference seen at base stations on all channels are recomputed.

Parameters used for simulation are listed in Table 1. $T_{H}$ and $\gamma_{t}$ are varied for different sets of simulations.

\section{Results and Analysis}

The system performance is measured by the average blocking and dropping probability within the total simulation time of $3600 \mathrm{~s}$.

$$
\begin{aligned}
& P_{b}=\text { new calls blocked/call arrivals } \\
& P_{d}=\text { dropped calls/accepted calls }
\end{aligned}
$$

Since dropping an existing call is much less desirable than blocking a new call, we also consider a weighted service denial rate $P_{b}+10 P_{d}$. Performance of handoff algorithm is measured by $E[H]$, mean number of handoffs/mobile, and $E\left[D_{\mathrm{ho}}\right]$, the average distance from old base station where a successful handoff is performed.

\subsection{Handoff Performance}

Figure 1 shows the probability of blocking plotted as a function of load in Erlang/Cell-Channel for three handoff algorithms: SIRBH, MPH and MPHT $(T=1)$. Results show that at traffic loads exceeding 0.4 Erlang/CellChannel, SIRBH blocks fewer calls than either MPH or MPHT. On the other hand, distinctions on the basis of call blocking are not so obvious at low load. Introducing a conditional timer to MPH algorithm does not result in any significant change in blocking probability. To keep

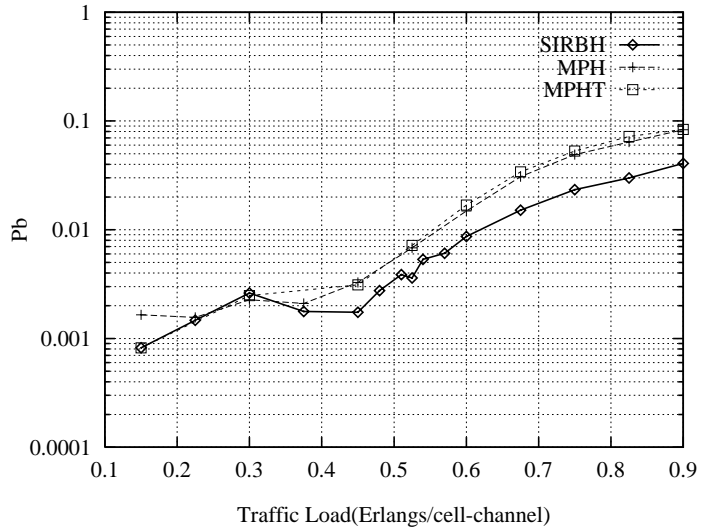

Figure 1: Blocking vs. Load $\left(\gamma_{\mathrm{drop}}=16 \mathrm{~dB}, \gamma_{t}=19 \mathrm{~dB}\right.$, $\gamma_{\text {new }}=22 \mathrm{~dB}$, OUTAGE $=0$ )

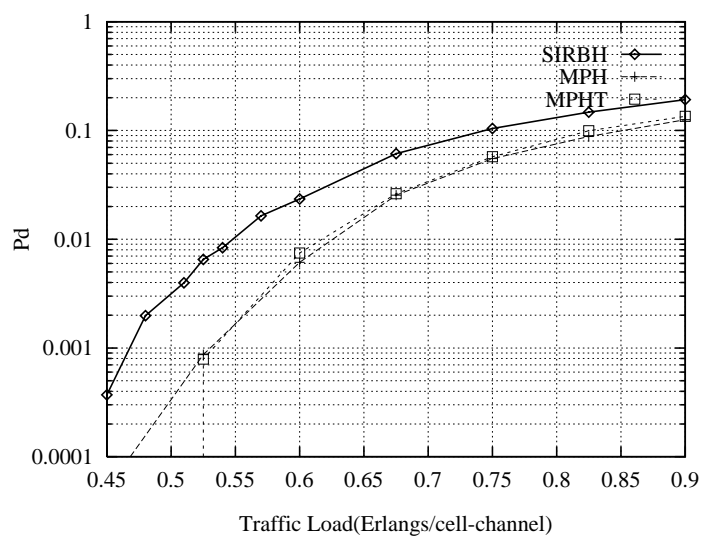

Figure 2: Dropping vs. Load $\left(\gamma_{\mathrm{drop}}=16 \mathrm{~dB}, \gamma_{t}=19 \mathrm{~dB}\right.$, $\gamma_{\text {new }}=22 \mathrm{~dB}$, OUTAGE $=0$ )

blocking probability below $1 \%$, both MPH and MPHT can admit 0.55 new calls/s to the system, while SIRBH can admit 0.62 new calls/s.

In Figure 2, we observe that the ability of SIRBH to admit more calls comes at the expense of higher call dropping. Both MPH and MPHT give better performance than SIRBH. MPH gives a slightly less dropping probability than MPHT but the difference is insignificant. MPH can carry an offered traffic load of 0.64 Erlang/Cell-Channel while keeping the dropping probability, $P_{d}$ at $1 \%$. With the same requirement for $P_{d}$, SIRBH permits 0.55 Erlang/CellChannel.

A combined performance index, $P_{b}+10 P_{d}$ is plotted in Figure 3. MPH and MPHT provide better grade of service than SIRBH. The number of dropped calls is higher in the case of SIRBH because handoffs occur only after the a mobile travels far into the adjacent cell until the SIR target $\gamma_{t}$ can not be maintained, even at power $p_{\max }$.

With an average velocity of $20 \mathrm{~m} / \mathrm{s}$, and a mean call du- 


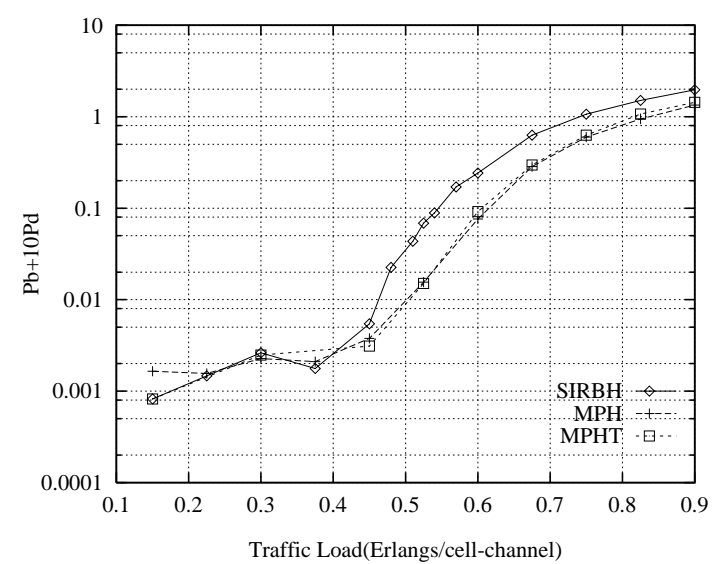

Figure 3: Combined Performance Measures $\left(\gamma_{\mathrm{drop}}=16 \mathrm{~dB}\right.$, $\gamma_{t}=19 \mathrm{~dB}, \gamma_{\text {new }}=22 \mathrm{~dB}$, OUTAGE $\left.=0\right)$

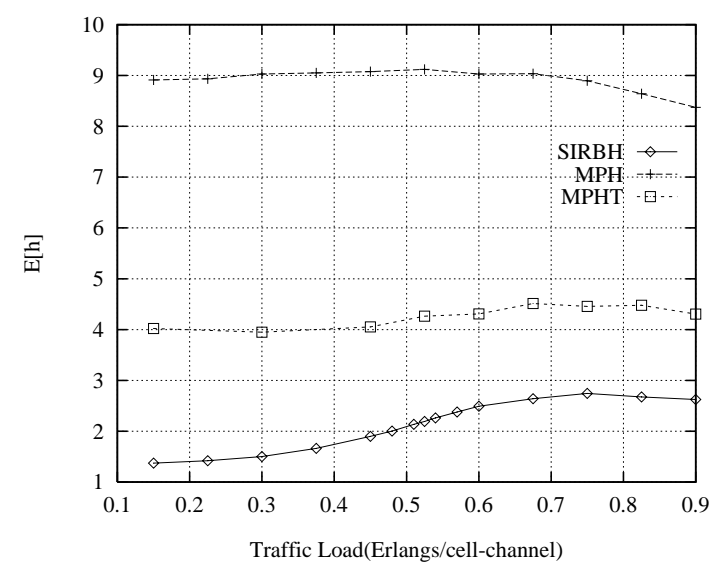

Figure 4: Mean Number of Handoffs per Call vs. Load $\left(\gamma_{\mathrm{drop}}=16 \mathrm{~dB}, \gamma_{t}=19 \mathrm{~dB}, \gamma_{\text {new }}=22 \mathrm{~dB}\right.$, OUTAGE $\left.=0\right)$

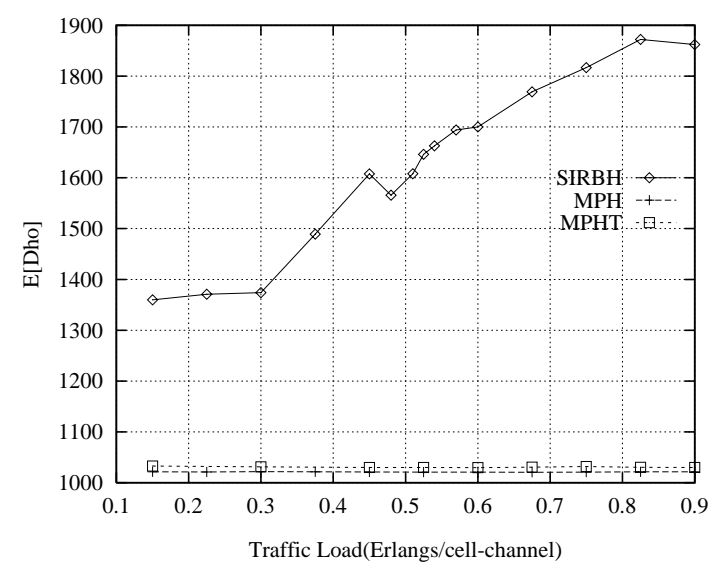

Figure 5: Mean Handoff Distance from Old Base Station as a Function of Traffic Load $\left(\gamma_{\mathrm{drop}}=16 \mathrm{~dB}, \gamma_{t}=19 \mathrm{~dB}\right.$, $\gamma_{\text {new }}=22 \mathrm{~dB}$, OUTAGE $=0$ )

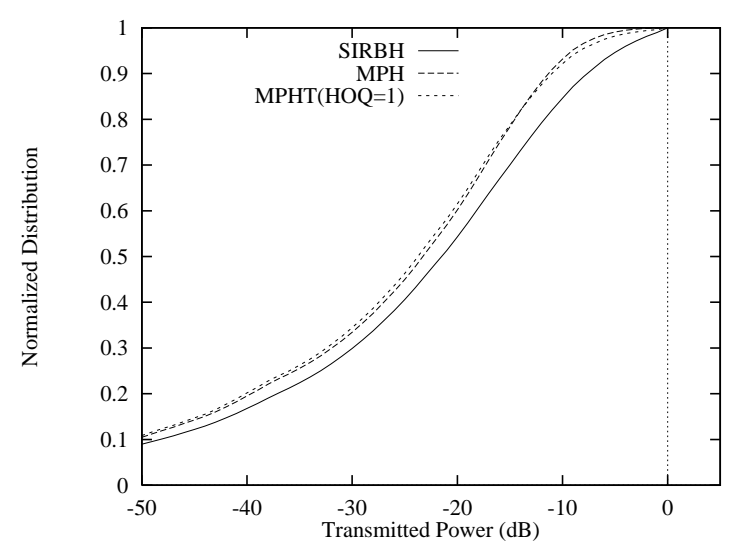

Figure 6: Normalized CDF of Power Transmitted by all users throughout the simulation $\left(\gamma_{\mathrm{drop}}=16 \mathrm{~dB}, \gamma_{t}=19 \mathrm{~dB}\right.$, $\gamma_{\text {new }}=22 \mathrm{~dB}$, OUTAGE $=0$ )

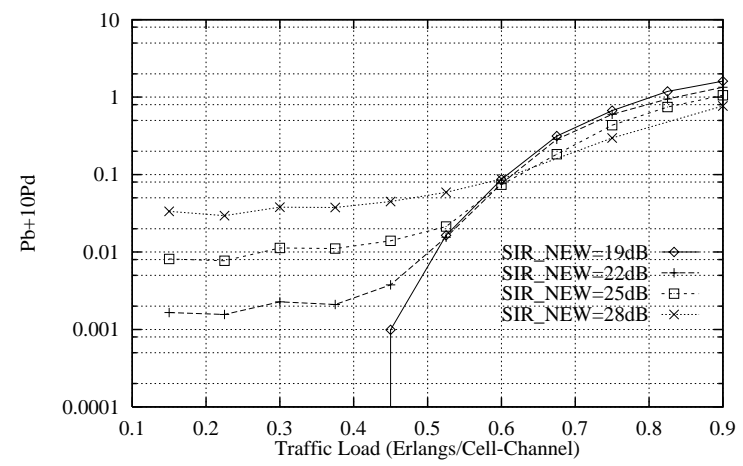

Figure 7: Combined Performance Measures for MPH as a function of $\gamma_{\text {new }}$ (SIR_NEW) $\left(\gamma_{\mathrm{drop}}=16 \mathrm{~dB}, \gamma_{t}=19 \mathrm{~dB}\right.$, OUTAGE $=0$ )

ration of $120 \mathrm{~s}$, a user travels $3 \mathrm{~km}$ on average during the call. For a cell size of $2 \mathrm{~km}$, this corresponds to 1.5 expected handoffs per call. Due to shadow fading and interference, the number of handoffs is usually somewhat greater. To better understand the handoff performance of these three algorithms, the mean number of handoffs/mobile $E[H]$ is plotted against traffic load in Figure 4. MPH without timer causes a large number of unnecessary handoffs, roughly 9 handoffs per call. The timer rule manages to reduce $E[H]$ to around 4 handoffs/call, but this is still higher than the 1.5 to 2.5 handoffs per call using the SIRBH algorithm

The average distance from the old base station to the point where an intercell handoff is performed is plotted in Figure 5. For both MPH and MPHT, the crossover distance stays level with respect to $\lambda$ in the range of $1020 \mathrm{~m}$, which implies that most handoffs happen at nominal cell boundary. On the other hand, the average crossover distance increases with load in SIRBH, ranging from 1400 to 
1900. SIRBH causes cell dragging, and handoffs are typically performed more than $400 \mathrm{~m}$ past the cell boundary.

Figure 6 show the normalized distribution of transmitter powers recorded throughout the simulation. Both MPH and MPHT use less power than SIRBH, with the cumulative distribution function (CDF) shifted about $4 \mathrm{~dB}$ to the left of that for SIRBH.

We conclude that both MPH and MPHT give better overall system performance than SIRBH. The drawback of MPH is the large number of unnecessary handoffs. This problem can be solved by introducing a timer. Results indicate that MPHT reduces $E[H]$ significantly without causing a noticeble increase in $P_{b}$ or $P_{d}$. Thus MPHT seems to be a promising solution.

\subsection{SIR Threshold Admission Control}

This section studies the effect of $\gamma_{\text {new }}$ based call admission policy on the system performance, with MPH being used in making handoff decisions. In Figure $7, P_{b}+10 P_{d}$ is plotted versus $\lambda$ as an index of overall system performance. Using a lower value for $\gamma_{\text {new }}$ gives better performance at $\lambda$ less than 0.6 Erlang/Cell-Channel, but the reverse is true for higher traffic load. This implies that when the system becomes congested, we need to restrict the number of new calls admitted so that handoff calls can be handled. Choosing a higher $\gamma_{\text {new }}$ helps to achieve this at a very high load. However, the SIR admission policy does not give significant improvement in system performance, and even results in unnecessarily high blocking in low traffic conditions.

Acknowledgement We gratefully acknowledge the constructive suggestions and discussions by the members of Radio Resource Allocation Group at WINLAB.

\section{References}

[1] Ray W. Nettleton, "Traffic Statistics in a Self-Organizing Cellular Telephone System", Proc. 40th IEEE Vehicular Technology Conference, pp. 305-310, May 1990.

[2] D. Hong, S. S. Rappaport, "Traffic Model and Performance Analysis for Cellular Mobile Radio Telephone Systems with Prioritized and Nonprioritized Handoff Procedures", IEEE Trans. Veh. Tech. vol VT-35, no 3, pp 77-92, Aug. 1986.

[3] W. R. Mende, "Evaluation Of A Proposed Handover Algorithm For The GSM Cellular System", Proc. 4oth IEEE Vehicular Technology Conference, pp. 264-269, May 1990.

[4] M. Gudmundson, "Analysis of Handover Algorithms in Cellular Radio Systems", Technical Report TRITA-TTT9107, Radio Communications Systems Lab., Royal Institute of Technology, Sweden.

[5] P. O. Gaasvik, M. Cornefjord, V. Svensson, "Different Methods of Giving Priority to Handoff Traffic in a Mobile Telephone System with Directed Retry", Proc. 41st
IEEE Vehicular Technology Conference, pp. 549-553, May 1991.

[6] M. Frodigh, "Optimum Dynamic Channel Allocation in Certain Street Microcellular Radio Systems", Proc. 42nd IEEE Vehicular Technology Conference., pp. 658-661, May 1992.

[7] S. S. Kuek, W. C. Wong, "Ordered Dynamic Channel Assignment Scheme with Reassignment in Highway Microcells", IEEE Trans. Veh. Tech., vol VT-41, no. 3, pp 271277, Aug. 1992.

[8] J. Zander, "Distributed cochannel interference control in cellular radio systems", IEEE Trans. Veh. Tech., vol. 41, no. 3, August 1992.

[9] S. A. Grandhi, R. Vijayan and D. J. Goodman, "A Distributed Algorithm for Power Control in Cellular Radio Systems", Thirtieth Annual Allerton Conference on Communication, Control, and Computing, Monticello, Illinois, September 30-October 2, 1992.

[10] D. J. Goodman, S. A. Grandhi, and R. Vijayan, "Distributed Dynamic Channel Assignment Schemes", Proc. 43rd IEEE Vehicular Technology Conference, pp. May, 1993.

[11] H. Xie, S. Kuek, "Priority Handoff Analysis", Proc. 43rd IEEE Vehicular Technology Conference, pp. 855-858, May 1993. May 1993.

[12] S. A. Grandhi, R. Vijayan, R. Yates, D.J. Goodman, and J.M. Holtzman, "Distributed Dynamic Resource Allocation", Technical Report WINLAB-TR58, Rutgers University, July 1993

[13] N. Zhang and J. M. Holtzman, "Analysis of Handoff Algorithms Using Both Absolute and Relative Measurements", IEEE Proc. Vehicular Technology Conference VTC-94, Stochkolm, Sweden, pp82-86, June 1994.

[14] R. D. Yates and C. Y. Huang, "Integrated power control and base station assignment", IEEE Transactions on Vehicular Technology, 1995. (To appear)

[15] R. D. Yates and C. Y. Huang, "Constrained Power Control and Base Station Assignment in Cellular Radio Systems", IEEE/ACM Transactions on Networking, 1995. (To appear)

[16] S.V. Hanly, "An Algorithm for Combined Cell-Site Selection and Power Control to Maximize Cellular Spread Spectrum Capacity", IEEE Journal on Selected Areas in Communications, 1995. (To appear)

[17] C. N. Chuah, R. D. Yates, D.J. Goodman, "Integrated Dynamic Radio Resource Management", 45th IEEE Vehicular Technology Conference \{to appear .

[18] S.A. Grandhi, J. Zander, R.D. Yates, "Constrained Power Control", Journal of Wireless Personal Communications. To appear. (Also available as TRITA-IT Report 94-06, Dept. of Teleinformation, Radio Commun. Systems Lab, Royal Institute of Technology, Sweden. June 1994.) 